\title{
Barreras y facilitadores para la investigación en ciencias de la salud durante la crisis del COVID-19:
} una revisión de alcance

\section{Health science research barriers and facilitators in the midst of the COVID-19 crisis: scoping review}

\author{
Karen Daniela Neira-Fernández ${ }^{1}$; Laura Gaitán-Lee²; Olga Janneth Gómez-Ramírez,
} MSc, PhD ${ }^{3}$

Recibido: 18 de noviembre de 2021/Aceptado: 15 de diciembre de 2021

\section{RESUMEN}

Objetivo: la pandemia ocasionada por el Covid-19 ha significado un gran desafío para la investigación en salud por la necesidad de dar una respuesta oportuna y efectiva a esta situación de crisis. Es importante proveer una visión panorámica sobre las principales barreras y facilitadores encontrados en la conducción de estudios en ciencias de la salud durante la crisis del Covid-19, así como también de las iniciativas en investigación sugeridas por autoridades en salud de investigación a nivel global, regional o local.

Materiales y métodos: se desarrolló una revisión sistemática de alcance. Se hizo una búsqueda de la literatura en Medline, Cochrane library, Lilacs y Google Scholar. Se incluyeron estudios de investigación originales, artículos de revisión, de opinión y editoriales disponibles en texto completo, publicados entre enero de 2020 y mayo de 2021 en español, inglés o portugués. Se hizo selección de

* Correspondencia: Karen Daniela Neira-Fernández. Dirección: Calle 20a \# 96c86. Teléfono: +57 3017272218. Correo electrónico: kdneiraf@unal.edu.co

1. Profesional en enfermería. Investigadora asociada. Instituto de Investigaciones Clínicas, Universidad Nacional de Colombia, Bogotá (Colombia).

2. Profesional en psicología. Investigadora asociada. Instituto de Investigaciones Clínicas, Universidad Nacional de Colombia, Bogotá (Colombia).

3. Enfermera. Profesora asociada. Universidad Nacional de Colombia, Bogotá (Colombia). los documentos y extracción de los datos por dos autores de manera independiente. Las barreras y facilitadores identificados fueron descritos y organizados en cuatro categorías a partir de la literatura: socioculturales, administrativos, organizacionales y metodológicos. Asimismo, se incluyeron documentos y comunicados oficiales de autoridades en salud e investigación a nivel global, regional y local. Los resultados se presentan de manera narrativa y en tablas.

Resultados: se seleccionaron 26 documentos para el análisis y síntesis de la información. Las barreras mencionadas más frecuentemente en la literatura incluyen las dificultades en cuanto al acceso a los participantes, a los trámites asociados a los comités de ética; así como el riesgo biológico para los investigadores y la falta de coordinación inter e intrainstitucional. Por su parte, los facilitadores identificados incluyen la adopción de soluciones virtuales, el trabajo cooperativo entre los actores de la investigación y la flexibilidad en el proceso de obtención del consentimiento informado. Frente a las iniciativas difundidas por las autoridades en salud e investigación, se identificaron cuatro estrategias relacionadas con la priorización de preguntas de investigación, el fomento de la cooperación y 
la inclusión en la investigación, la lucha contra la infodemia y el fortalecimiento de la calidad metodológica de los estudios.

Conclusiones: para la investigación en el contexto de la pandemia representa un desafío continuar con la cooperación e interoperabilidad entre las instituciones, los países y las disciplinas, con el fin de facilitar los procesos investigativos en el futuro; del mismo modo, cobra importancia mantener la ciencia abierta y la financiación de estudios cooperativos cuando surjan otras prioridades. Asimismo, es evidente la necesidad de desarrollar y sostener mecanismos que gestionen la información de manera eficiente para la toma de decisiones. Se requiere evaluar de manera continua los efectos que ha dejado esta pandemia en la práctica de la investigación en ciencias de la salud para comprender de manera integral lo que debemos aprender como sociedad a partir de las crisis.

Palabras clave: COVID-19, investigación cualitativa, ciencias de la salud, estudios clínicos como asunto.

\section{ABSTRACT}

Objective: The COVID-19 pandemic has imposed a great challenge on health research because of the pressing need to respond promptly and effectively to this crisis situation. It is important to offer a high level perspective of the main barriers and facilitators found when conducting health science studies during the COVID-19 crisis and to discuss the research initiatives suggested by global, regional or local health research authorities.

Materials and methods: A systematic scoping review was carried out. A literature search was conducted in the Medline, Cochrane Library, Lilacs and Google Scholar databases. Original research studies, review and opinion articles and editorials available in full text published in Spanish, English or Portuguese between January 2020 and May 2021 were included. Two authors working independently selected the papers and collected the data. The barriers and facilitators identified were described and organized in four categories according to the literature: sociocultural, administrative, organizational and methodological. Official documents and communications from global, regional and local health and research authorities were also included. Results are presented in narrative and table form. Results: Overall, 26 documents were selected for data analysis and synthesis. The barriers most frequently mentioned in the literature include issues with access to participants, ethics committees paperwork, biological risk for the researchers, and lack of inter and intra-institutional coordination. On the other hand, the facilitators identified include the adoption of on-line solutions, cooperative work among research actors, and a more flexible informed consent process. Regarding the initiatives disseminated by health and research authorities, four strategies were identified: prioritization of research questions, cooperation and inclusion promotion, fight against infodemia, and strengthening of the methodological quality of the studies.

Conclusions: Continued cooperation and interoperability among institutions, countries and disciplines to facilitate future research processes is a challenge in the context of the pandemic; similarly, it is now important to maintain open science and funding of cooperative studies when other priorities emerge. Likewise, there is an evident need to develop and sustain efficient information management systems to help with decision-making. Ongoing review of the effects of the pandemic on health research practice is needed in order to gain comprehensive insights of what we need to learn as a society from this crisis.

Keywords: COVID-19, qualitative research, health sciences, clinical studies as topic.

\section{INTRODUCCIÓN}

La infección por el virus Cov2 fue declarada pandemia en marzo de 2020 por parte de la Organización Mundial de la Salud (OMS) (1). Produce la condición conocida como el Síndrome Agudo Respiratorio Severo (SARS) Covid-19 (2) que se asocia con importante morbilidad y mortalidad (3). La infección ha impactado a nivel social, económico, 
político y sanitario tanto a los países de altos ingresos, como a los países de medianos y bajos ingresos, entre ellos, los países de Latinoamérica (4). El impacto económico del Covid-19, de acuerdo con el Banco Mundial, tuvo que ver con la adopción de medidas para su contención, la cual ocasionó una reducción de más del 7\% de la economía para Latinoamérica y el Caribe. Dicho impacto desestabilizó todos los sectores, teniendo grandes repercusiones en el financiamiento y manejo de recursos en el ámbito investigativo (4).

Por ser una infección no conocida hasta su aparición, el SARS Covid-19 representó un desafío particular para la investigación. La ciencia y la academia fueron llamadas a responder de manera oportuna y adecuada a los retos y las incógnitas planteadas por el nuevo virus (5). Por todo esto, durante el 2020 y el 2021 se generó a nivel internacional una cantidad importante de literatura científica sobre la epidemiología, fisiopatología, métodos diagnósticos, intervenciones preventivas y tratamientos para el SARS, como también respecto a la experiencia de investigar dentro de este contexto (6).

Es importante registrar cómo se desarrolló la investigación en ciencias de la salud durante esta pandemia, en aspectos tales como las preguntas a contestar, las innovaciones metodológicas para obtener respuestas validas de manera rápida, cuáles fueron las barreras que dificultaron los procesos investigativos, y los aspectos que lo facilitaron, o cuál fue la política, las iniciativas sugeridas por organismos multilaterales o locales de investigación en salud, la magnitud de la inversión en investigación global por regiones o por país de acuerdo con las diferencias en desarrollo tecnológico entre los países de altos, medios y bajos ingresos (7).

De esta manera, nuestro objetivo fue desarrollar una revisión de la literatura internacional para dar al lector una visión panorámica sobre las principales barreras y facilitadores encontrados en la conducción de estudios durante la crisis del Covid-19, así como también describir las iniciativas y directrices difundidas por organizaciones multilaterales y nacionales de salud.

\section{MATERIALES Y MÉTODOS}

Diseño. Revisión sistemática de alcance (RSA). Se siguió la metodología del Instituto Johana Briggs (8) para el desarrollo de este tipo de revisiones. La pregunta de investigación de esta revisión se planteó de la siguiente forma bajo el acrónimo PCC (población, concepto y contexto): ¿Cuáles son las barreras y facilitadores (concepto) para la investigación en ciencias de la salud (población) que reportó la literatura durante la crisis del Covid-19 (contexto)? Como pregunta auxiliar se buscó mapear: ¿Cuáles son las iniciativas propuestas por organismos internacionales o nacionales de salud en investigación? Criterios de elegibilidad de la literatura. Se incluyeron estudios de investigación originales, (estudios cualitativos y cuantitativos con datos primarios), artículos de revisión, de opinión y editoriales que reportaron el concepto de las barreras y facilitadores percibidos durante el proceso de formulación, aprobación, conducción, análisis y publicación de estudios desarrollados en el área de ciencias de la salud durante la pandemia por Covid-19.

Fueron incluidos documentos publicados desde diciembre del 2019 (época del descubrimiento del virus) hasta mayo del 2021 en idioma español, inglés o portugués. Se excluyeron los documentos publicados únicamente en formato de resumen o que no reportaran el proceso investigativo en el contexto de la pandemia por el COVID 19.

Estrategias de búsqueda. Se realizó una búsqueda de evidencia disponible a través de las bases electrónicas Medline, Cochrane library, Lilacs y Google scholar, mediante términos controlados MESH y DeCS, enlazados a términos de texto libre de "coronavirus", "coronavirus disease 2019", "coronavirus infections", "COVID19 virus", "cov19”, “2019-ncov”, “covid-19 pandemic”, "clinical research", "clinical study", "clinical trial”, “ensayos clínicos", "barriers", “obstacles”, “challenges”, "difficulties", "facilitators", "enablers", "barreras" y "facilitadores". Los términos fueron combinados a conveniencia mediante los operadores booleanos (AND, OR NOT), obteniendo las ecuaciones de búsqueda a usar en las bases de datos. Para cada bús- 
queda se generó una bitácora con el fin de garantizar su reproducibilidad y transparencia (ver Anexo 1). Finalmente, se hizo uso del sistema "bola de nieve" con las fuentes obtenidas para complementar el paso anterior e identificar un mayor número de referencias concernientes al tema. La gestión del listado de las referencias bibliográficas identificadas en las búsquedas electrónicas se realizó en el programa Rayyan (9), en donde se eliminaron las publicaciones duplicadas. Por otra parte, para la búsqueda de los principales comunicados de las organizaciones multilaterales o locales en salud en respuesta a la pandemia se hizo una revisión de sus canales web.

Selección de fuentes de evidencia. Se llevó a cabo una tamización inicial entre las investigadoras de manera independiente (DNF y LGL), teniendo en cuenta el título de la publicación y el resumen; posteriormente, se aplicaron los criterios de inclusión y exclusión para cada manuscrito evaluado. A partir del grupo de referencias preseleccionadas, se realizó la selección de estudios por texto completo. Los casos de conflicto o discrepancia fueron resueltos mediante consenso entre los revisores.

Extracción de datos. Se extrajeron de manera independiente por dos investigadoras (DNF y LGL) las siguientes variables: autor; país de realización; año de publicación; tipo de estudio o documento; barreras identificadas; facilitadores. En cuanto a los documentos de las organizaciones de salud, se extrajo información sobre la entidad que las propone, tipo de iniciativa o políticas en investigación propuestas, así como las recomendaciones a futuro.

Análisis de la evidencia. El informe de esta revisión se realizó conforme a la declaración de ítems de referencia para publicar revisiones sistemáticas y metaanálisis y su extensión para revisiones de alcance (PRISMA-ScR, por sus siglas en inglés) (10).

Primero se llevó a cabo un análisis descriptivo de los hallazgos en donde se categorizó temáticamente el contenido y a partir de los datos se construyeron subcategorías que permitieron presentar los resultados de una manera más clara.

Se describen las iniciativas difundidas por diversas organizaciones multilaterales regionales y locales. En la discusión se hace un análisis de la aplicabilidad entre las iniciativas difundidas por las principales autoridades en salud e investigación en relación con las barreras y facilitadores encontradas en la literatura académica.

\section{RESULTADOS}

Búsqueda y selección de los documentos. Inicialmente se identificaron 1013 títulos, y se descartaron 5 documentos después de eliminar duplicados; tras la lectura del título y resumen, se descartaron 959 documentos que no cumplieron con los criterios de elegibilidad propuestos y, de este modo, quedaron un total de 49 documentos que fueron leídos en texto completo, de los cuales 23 documentos no cumplieron con los criterios de población, concepto y contexto; finalmente, incluyeron 26 artículos para realizar el análisis final y síntesis de la información (ver Figura 1).

Figura 1.

Diagrama de flujo del proceso de selección de artículos

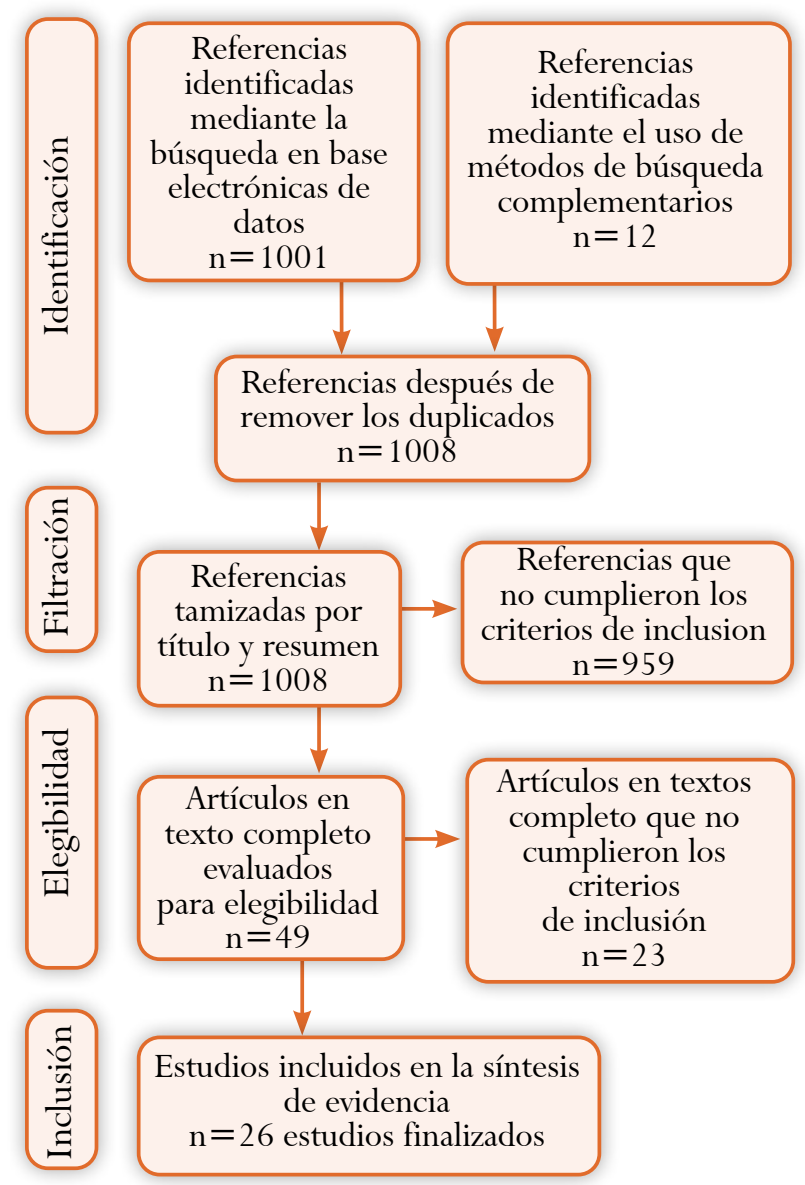


Características de los documentos incluidos. En términos del lugar de origen de los autores de los documentos se encontró que doce de ellos eran provenientes de Norteamérica: nueve de los Estados Unidos (11-19) y tres de Canadá (20-22). China aporta dos $(23,24)$, y Latinoamérica dos referencias $(6,25)$; Europa aportó 4 documentos, dos de Francia $(26,27)$, uno de Italia (28) y otro de Reino Unido (29); Asia aporta siete documentos: dos de China previamente mencionados, dos de la India $(30,31)$, un documento de Jordán (32), uno de Emiratos Árabes (33) y uno de Filipinas (34). Finalmente, África tuvo un documento proveniente de Sudáfrica (35). Respecto al tipo de documento, 19 corresponden a comentarios, editoriales o columnas de opinión (11-14,16-19,21-26,28,30,31,34,35), 5 son revisiones de literatura $(15,20,29,32,33)$ y los dos restantes son estudios de investigación original, con una metodología claramente delimitada y replicable $(6,27)$ (ver Tabla 1).

\begin{tabular}{|c|c|c|c|c|c|}
\hline \multicolumn{6}{|c|}{$\begin{array}{c}\text { Tabla } 1 . \\
\text { Características de los estudios incluidos en la revisión. }\end{array}$} \\
\hline Autor/año & País & $\begin{array}{l}\text { Tipo de } \\
\text { documento }\end{array}$ & $\begin{array}{l}\text { Autodenominación } \\
\text { del documento }\end{array}$ & Contexto & Concepto \\
\hline $\begin{array}{l}\text { Hashem } \\
\text { et al, } 2020 \text {. }\end{array}$ & Jordania & $\begin{array}{l}\text { Revisión de } \\
\text { literatura }\end{array}$ & Artículo de revisión & Global & $\begin{array}{l}\text { Ensayos clínicos en busca de } \\
\text { tratamientos para el manejo del } \\
\text { SARS por Covid- } 19 \text {. }\end{array}$ \\
\hline $\begin{array}{l}\text { Madariaga } \\
\text { et al, } 2021 .\end{array}$ & Canadá & $\begin{array}{l}\text { Revisión de } \\
\text { literatura }\end{array}$ & $\begin{array}{l}\text { Revisión de la } \\
\text { literatura a pequeña } \\
\text { escala }\end{array}$ & Global & $\begin{array}{l}\text { Retos de la investigación clínica } \\
\text { durante la pandemia. }\end{array}$ \\
\hline $\begin{array}{l}\text { Verna } \\
\text { et al, } 2020 \text {. }\end{array}$ & EE. UU. & Comentario & Artículo especial & Global & $\begin{array}{l}\text { Investigación clínica no } \\
\text { Covid-19 durante la pandemia } \\
\text { (Investigación en hepatología). }\end{array}$ \\
\hline $\begin{array}{l}\text { AlNaamani } \\
\text { et al, } 2020 .\end{array}$ & $\begin{array}{l}\text { Oman/ } \\
\text { Canadá }\end{array}$ & Comentario & Revisión de opinión & Global & $\begin{array}{l}\text { Ensayos clínicos y estudios } \\
\text { observacionales que inician } \\
\text { previo durante y posterior a la } \\
\text { pandemia. }\end{array}$ \\
\hline $\begin{array}{l}\text { Cagnazzo } \\
\text { et al, } 2021 \text {. }\end{array}$ & Italia & Comentario & Editorial & Local & $\begin{array}{l}\text { Retos de la investigación clínica } \\
\text { durante la pandemia. }\end{array}$ \\
\hline $\begin{array}{l}\text { Carracedo } \\
\text { et al, } 2021 \text {. }\end{array}$ & $\mathrm{N} / \mathrm{A}$ & $\begin{array}{l}\text { Estudio de } \\
\text { investigación } \\
\text { original }\end{array}$ & $\begin{array}{l}\text { Estudio de } \\
\text { investigación original }\end{array}$ & Regional & $\begin{array}{l}\text { Ensayos clínicos en busca de } \\
\text { tratamientos para el manejo del } \\
\text { SARS por Covid- } 19 \text {. }\end{array}$ \\
\hline $\begin{array}{l}\text { González- } \\
\text { Duarte } \\
\text { et al, } 2020 \text {. }\end{array}$ & México & Comentario & No reporta & Global & $\begin{array}{l}\text { Aspectos éticos para las } \\
\text { investigaciones clínicas y } \\
\text { observacionales en tiempos de } \\
\text { pandemia. }\end{array}$ \\
\hline $\begin{array}{l}\text { Meyer } \\
\text { et al, } 2021 \text {. }\end{array}$ & EE. UU. & $\begin{array}{l}\text { Comentario } \\
\text { (reunión de } \\
\text { expertos) }\end{array}$ & No reporta & Global & $\begin{array}{l}\text { Ensayos clínicos en busca de } \\
\text { tratamientos para el manejo del } \\
\text { SARS por Covid- } 19 \text {. }\end{array}$ \\
\hline $\begin{array}{c}\text { Dron } \\
\text { et al, } 2021 .\end{array}$ & $\begin{array}{c}\text { UK/ } \\
\text { Canadá }\end{array}$ & Comentario & Artículo de opinión & Global & $\begin{array}{l}\text { Ensayos clínicos en busca de } \\
\text { tratamientos para el manejo del } \\
\text { SARS por Covid- } 19 \text {. }\end{array}$ \\
\hline $\begin{array}{c}\text { Bahans } \\
\text { et al } 2021 .\end{array}$ & Francia & Comentario & No reporta & Local & $\begin{array}{l}\text { Aspectos éticos para las } \\
\text { investigaciones clínicas y } \\
\text { observacionales en tiempos de } \\
\text { pandemia. }\end{array}$ \\
\hline $\begin{array}{c}\text { Yang } \\
\text { et al, } 2020 \text {. }\end{array}$ & China & Comentario & Revisión de opinión & Local & \\
\hline
\end{tabular}




\begin{tabular}{|c|c|c|c|c|c|}
\hline Autor/año & País & $\begin{array}{c}\text { Tipo de } \\
\text { documento }\end{array}$ & $\begin{array}{l}\text { Autodenominación } \\
\text { del documento }\end{array}$ & Contexto & Concepto \\
\hline $\begin{array}{l}\text { Bhatt } \\
2020 .\end{array}$ & India & Comentario & Revisión de opinión & Global & $\begin{array}{l}\text { Ensayos clínicos en busca de } \\
\text { tratamientos para el manejo del } \\
\text { SARS por Covid- } 19 \text {. }\end{array}$ \\
\hline $\begin{array}{c}\text { Becker } \\
\text { et al, } 2021 .\end{array}$ & Francia & $\begin{array}{l}\text { Estudio de } \\
\text { investigación } \\
\text { original }\end{array}$ & $\begin{array}{c}\text { Estudio de } \\
\text { investigación original }\end{array}$ & Local & $\begin{array}{l}\text { Ensayos clínicos en busca de } \\
\text { tratamientos para el manejo del } \\
\text { SARS por Covid- } 19 \text {. }\end{array}$ \\
\hline $\begin{array}{l}\text { Ibrahim } \\
\text { et al, } 2020 \text {. }\end{array}$ & $\begin{array}{l}\text { Emiratos } \\
\text { Árabes } \\
\text { Unidos }\end{array}$ & $\begin{array}{l}\text { Revisión de } \\
\text { literatura }\end{array}$ & Artículo de revisión & Regional & $\begin{array}{l}\text { Investigación clínica no } \\
\text { Covid-19 durante la pandemia. }\end{array}$ \\
\hline $\begin{array}{l}\text { Mourad } \\
\text { et al, } 2020 .\end{array}$ & EE. UU. & Comentario & No reporta & Local & $\begin{array}{l}\text { Ensayos clínicos en busca de } \\
\text { tratamientos para el manejo del } \\
\text { SARS por Covid- } 19 \text {. }\end{array}$ \\
\hline $\begin{array}{c}\text { Bierer } \\
\text { et al, } 2020 \text {. }\end{array}$ & EE. UU. & Comentario & No reporta & Global & $\begin{array}{l}\text { Investigación clínica no } \\
\text { Covid-19 durante la pandemia } \\
\text { (investigación perinatal). }\end{array}$ \\
\hline $\begin{array}{l}\text { Marcum } \\
\text { et al, } 2020 .\end{array}$ & EE. UU. & $\begin{array}{l}\text { Revisión de } \\
\text { literatura }\end{array}$ & Artículo de revisión & Local & $\begin{array}{l}\text { Aspectos éticos para las } \\
\text { investigaciones clínicas y } \\
\text { observacionales en tiempos de } \\
\text { pandemia. }\end{array}$ \\
\hline $\begin{array}{l}\text { Almufleh } \\
\text { et al, } 2021 \text {. }\end{array}$ & EE. UU. & Comentario & Comentario & Global & $\begin{array}{l}\text { Investigación clínica no } \\
\text { Covid-19 durante la pandemia } \\
\text { (Investigación Oncológica). }\end{array}$ \\
\hline $\begin{array}{l}\text { Sohrabi } \\
\text { et al, } 2021 \text {. }\end{array}$ & UK & $\begin{array}{l}\text { Revisión de } \\
\text { literatura }\end{array}$ & Artículo de revisión & Global & $\begin{array}{l}\text { Ensayos clínicos pragmáticos } \\
\text { como alternativa para generar } \\
\text { oportunamente la evidencia } \\
\text { clínica necesaria en la pandemia. }\end{array}$ \\
\hline $\begin{array}{l}\text { Singh } \\
\text { et al, } 2020 \text {. }\end{array}$ & $\begin{array}{l}\text { India/ } \\
\text { Kenia }\end{array}$ & Comentario & No reporta & Global & $\begin{array}{l}\text { Revisión integral y evaluación } \\
\text { crítica del impacto de la } \\
\text { pandemia sobre investigación } \\
\text { científica y financiamiento. }\end{array}$ \\
\hline $\begin{array}{l}\text { Ma et al, } \\
2020 .\end{array}$ & China & Comentario & Comentario & Global & $\begin{array}{l}\text { Investigación clínica no } \\
\text { Covid-19 durante la pandemia. }\end{array}$ \\
\hline $\begin{array}{c}\text { Perez } \\
\text { et al, } 2020 \text {. }\end{array}$ & EE. UU. & Comentario & Comentario & Global & $\begin{array}{l}\text { Ensayos clínicos en busca de } \\
\text { tratamientos para el manejo del } \\
\text { SARS por Covid- } 19 \text {. }\end{array}$ \\
\hline $\begin{array}{c}\text { Weiner } \\
\text { et al, } 2020 .\end{array}$ & EE. UU. & Comentario & Comentario & Global & $\begin{array}{l}\text { Retos de la investigación clínica } \\
\text { durante la pandemia. }\end{array}$ \\
\hline $\begin{array}{c}\text { Pepper } \\
\text { et al, } 2020 .\end{array}$ & Sudáfrica & Comentario & No reporta & Global & $\begin{array}{l}\text { Investigación clínica no } \\
\text { Covid-19 durante la pandemia. }\end{array}$ \\
\hline $\begin{array}{l}\text { Reyes, } \\
2020 .\end{array}$ & Filipinas & Comentario & Artículo destacado & Local & $\begin{array}{l}\text { Retos de la investigación clínica } \\
\text { durante la pandemia. }\end{array}$ \\
\hline $\begin{array}{l}\text { Saberi, } \\
2020 .\end{array}$ & EE. UU. & Comentario & Notas de campo & Global & $\begin{array}{l}\text { Aspectos éticos para las } \\
\text { investigaciones clínicas y } \\
\text { observacionales en tiempos de } \\
\text { pandemia. }\end{array}$ \\
\hline
\end{tabular}

Continuación Tabla 1

1 Comentario hace referencia a documentos de corte editorial que evidencia la experiencia, perspectiva o reflexión del autor sobre un tema en particular, respaldada por la literatura.

2 Revisión de literatura hace referencia a un sondeo y análisis de las investigaciones publicadas anteriormente sobre el tema.

3 Estudio de investigación original hace referencia a estudios con una metodología claramente delineada y replicable. 
Tópico del documento. En 17 documentos se discute la investigación en pandemia a nivel global $(11,12,14,16-22,24,25,29-32,35)$, dos a nivel regional $(6,33)$ y siete a nivel local $(13,15,23,26-28,34)$. Finalmente, en cuanto a los tópicos trabajados, se encontró que ocho hablaban sobre barreras y facilitadores dentro de ensayos clínicos en busca de tratamientos para el manejo de SARS por covid-19 (6,12,22-24,31-33), seis documentos reflexionaban sobre la investigación en ciencias de la salud no relacionada con COVID; en el contexto de pandemia $(11,13,15,18,27,30)$, cinco tratan sobre diversos tipos de investigación clínica durante la pandemia $(17,19,20,28,35)$, cuatro discutían aspectos éticos para las investigaciones clínicas y observacionales en tiempos de pandemia $(14,25,26,34)$, y los tres manuscritos restantes discutían algunas variaciones de estos tópicos principales que fueron lo suficientemente diferentes para no poderse categorizar juntos $(16,21,29)$.

Para describir de manera conceptual las barreras y los facilitadores se hizo uso de los tipos de barreras en salud descritos por Caicedo-Rosero y EstradaMontoya (36), a saber: barreras socioculturales, administrativas y organizacionales. Se creó adicionalmente una categoría que tuviera en cuenta las barreras metodológicas particulares al proceso de investigación en ciencias de la salud que no se contemplaban en las categorías recién descritas. Luego se realizó el mismo proceso para los facilitadores.

Barreras. Dentro de los aspectos socioculturales resaltó particularmente la subcategoría de dificultades de acceso a los participantes, como por ejemplo, poblaciones que no tienen acceso a medios electrónicos para participar de manera remota en estudios. Esta barrera es mencionada en nueve documentos $(11,15-17,19,21,22,27,30)$.

En la categoría de aspectos administrativos, se encontró que las barreras que más se mencionaron fueron las dificultades asociadas a comités de ética en 11 documentos, entre ellas destacan el incremento en el flujo de trabajo, el cual excede la capacidad de los comités; la diversidad de criterios y estándares exigidos por comités de diferentes or- ganizaciones; la escasez de guías y directrices que orientarán la toma de decisiones en un contexto de emergencia en salud pública; las implicaciones de la obtención del consentimiento informado con participantes en estados vulnerables y el riesgo de contagio que representa la firma presencial del documento $(12,14,16,20,24-26,28,31,32,34)$, y los recursos limitados para su funcionamiento en ocho documentos $(6,12-14,20,22,32,34)$. Otra barrera organizacional es el riesgo de contagio de los investigadores con el consecuente efecto sobre el equipo de investigación, la dificultad para mantenerlos contratados a plazo fijo o el reemplazo de estos por personal menos capacitado están dificultando la correcta ejecución de estudios (11,20,28-30,32), esto agregado a la escasez de equipos de protección personal y otros factores como la pérdida de activos en la investigación $(13,18,29)$.

A nivel organizacional y de logística, nueve estudios destacaron la falta de coordinación inter e intrainstitucional $(6,12,14,16,25,26,30,32,33)$, siete la competencia por reclutamiento $(6,12,22,23,25,26,32)$, y siete los riesgos ocupacionales del equipo $(11,13,15,20,29,32,35)$. Un gran número de documentos demuestran que existen dificultades en la coordinación y colaboración entre estados e instituciones para lograr estudios colaborativos más grandes, tales como la falta de estandarización de las lógicas de trabajo o las dificultades en la adherencia a protocolos por diferencias en leyes y regulaciones, por lo cual resulta más viable hacer investigaciones pequeñas que terminan siendo estudios duplicados o con poco poder estadístico $(6,16,20,32)$. Los hallazgos dan muestra de que hay muy pocos estudios colaborativos y poca priorización de estos (26). Finalmente, dentro de la categoría de aspectos metodológicos la principal barrera identificada fue la obtención del tamaño de la muestra, mencionada en ocho estudios $(6,16,17,22,26,31,34)$. La construcción de este sistema categorial de barreras y sus respectivas subcategorías se presentan de manera detallada en la Tabla 2. 


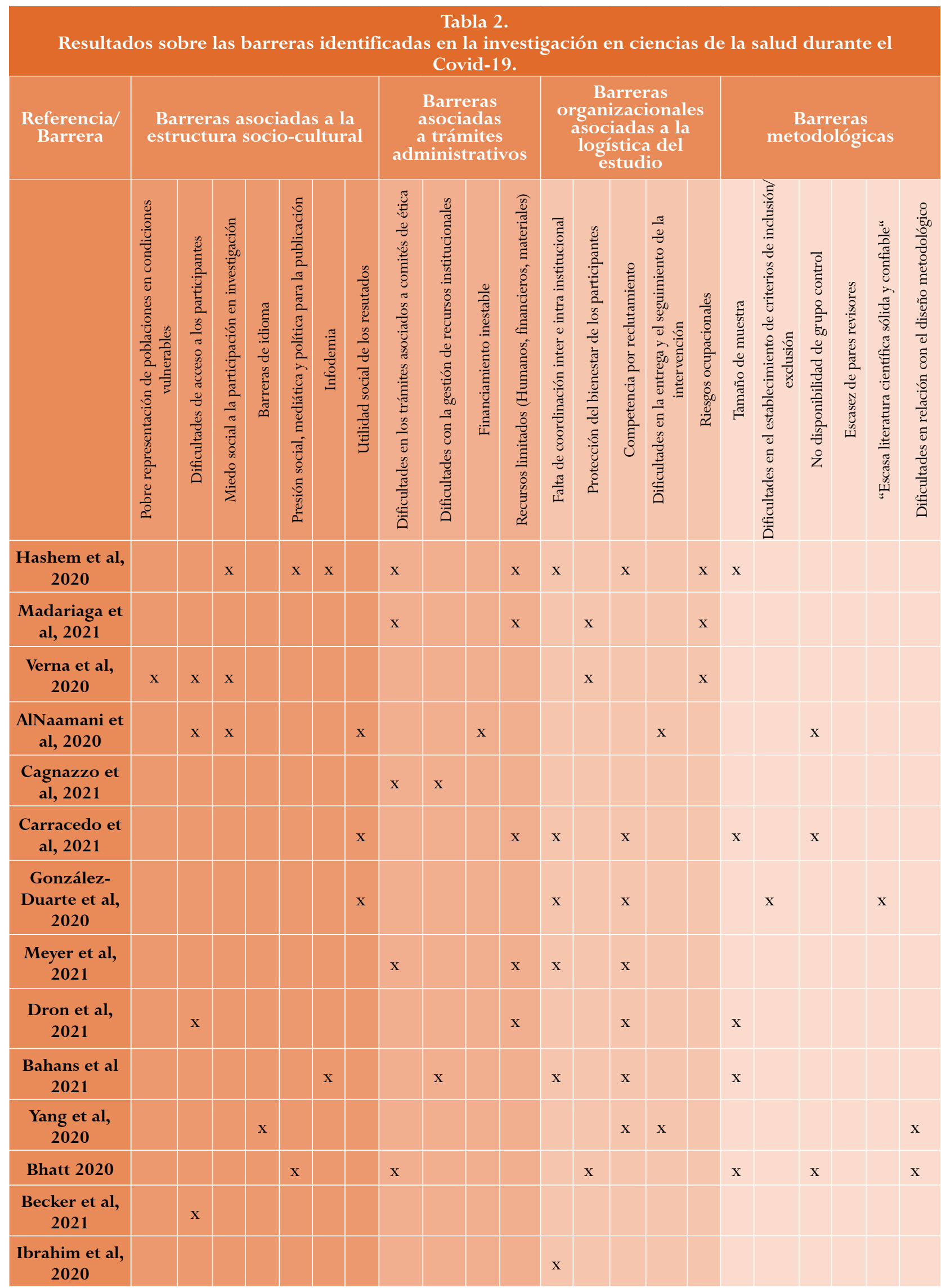




\begin{tabular}{|c|c|c|c|c|c|c|c|c|c|c|c|c|c|c|c|c|c|c|c|c|c|c|}
\hline \multirow[t]{2}{*}{$\begin{array}{l}\text { Referencia/ } \\
\text { Barrera }\end{array}$} & \multicolumn{7}{|c|}{$\begin{array}{l}\text { Barreras asociadas a la } \\
\text { estructura socio-cultural }\end{array}$} & \multicolumn{4}{|c|}{$\begin{array}{c}\text { Barreras } \\
\text { asociadas } \\
\text { a trámites } \\
\text { administrativos }\end{array}$} & \multicolumn{5}{|c|}{$\begin{array}{c}\text { Barreras } \\
\text { organizacionales } \\
\text { asociadas a la } \\
\text { logística del } \\
\text { estudio }\end{array}$} & \multicolumn{6}{|c|}{$\begin{array}{l}\text { Barreras } \\
\text { metodológicas }\end{array}$} \\
\hline & 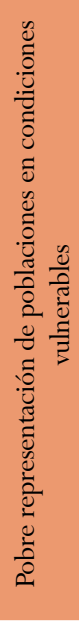 & 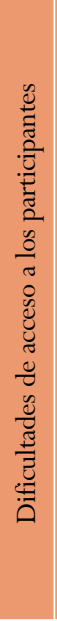 & 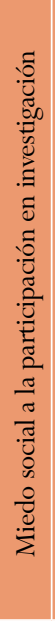 & 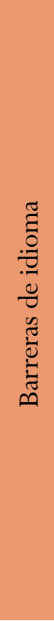 & 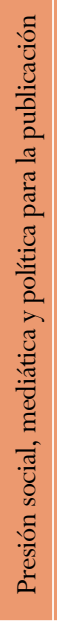 & 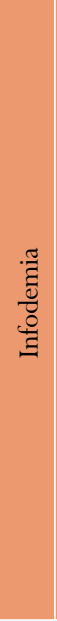 & 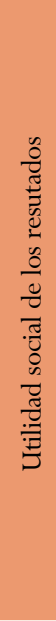 & 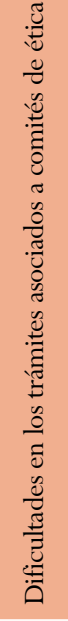 & 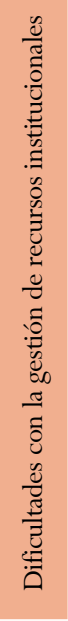 & 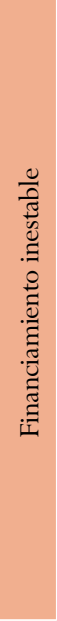 & 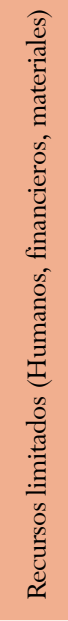 & 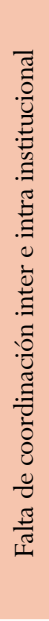 & 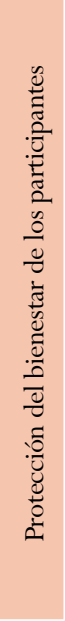 & 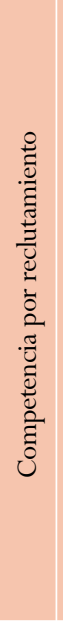 & 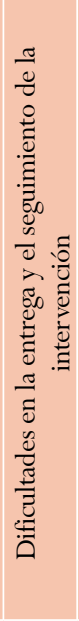 & 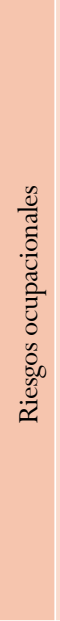 & 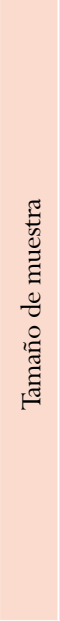 & 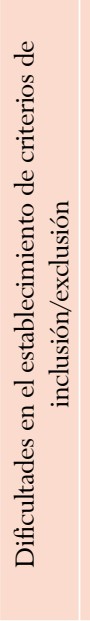 & 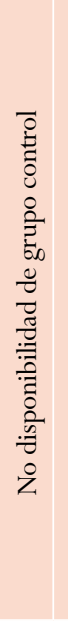 & 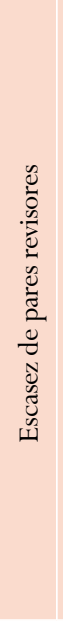 & 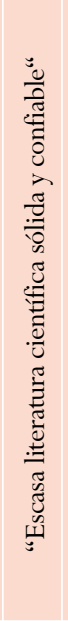 & 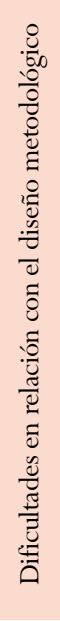 \\
\hline $\begin{array}{l}\text { Mourad et al, } \\
2020\end{array}$ & & & & & & & & & & & $\mathrm{x}$ & & $\mathrm{x}$ & & & $\mathrm{x}$ & & & & & & \\
\hline $\begin{array}{l}\text { Bierer et al, } \\
2020\end{array}$ & & & & & & & & $\mathrm{x}$ & & & $\mathrm{x}$ & $\mathrm{x}$ & & & & & & & & & & \\
\hline $\begin{array}{l}\text { Marcum et } \\
\text { al, } 2020\end{array}$ & & $\mathrm{x}$ & & & & & & & & $\mathrm{x}$ & & & $\mathrm{x}$ & & & $\mathrm{x}$ & & & & & & \\
\hline $\begin{array}{l}\text { Almufleh et } \\
\text { al, } 2021\end{array}$ & & $\mathrm{x}$ & $\mathrm{x}$ & & & & & $\mathrm{x}$ & & $\mathrm{x}$ & & $\mathrm{x}$ & & & & & $\mathrm{x}$ & & & & & \\
\hline $\begin{array}{l}\text { Sohrabi et al, } \\
2021\end{array}$ & & & & & & $\mathrm{x}$ & & & & & & & & & & $\mathrm{x}$ & & & & & & \\
\hline $\begin{array}{l}\text { Singh et al, } \\
2020\end{array}$ & & $\mathrm{x}$ & & & & & & & & & & $\mathrm{x}$ & & & $\mathrm{x}$ & & & & & & & \\
\hline $\begin{array}{c}\text { Ma et al, } \\
2020\end{array}$ & & & & & & & & $\mathrm{x}$ & & & & & & & & & & & & & & \\
\hline $\begin{array}{c}\text { Perez et al, } \\
2020\end{array}$ & & $\mathrm{x}$ & & & & & & & & & & & & & $\mathrm{x}$ & & $\mathrm{x}$ & & & & & \\
\hline $\begin{array}{l}\text { Weiner et al, } \\
2020\end{array}$ & & & & & & & & & & & & & & & $\mathrm{x}$ & & & & & $\mathrm{x}$ & & \\
\hline $\begin{array}{l}\text { Pepper et al, } \\
2020\end{array}$ & & & & & $\mathrm{x}$ & & & & $\mathrm{x}$ & & & & & & & $\mathrm{x}$ & & & & & & \\
\hline Reyes, 2020 & & & & & & & & $\mathrm{x}$ & & & $\mathrm{x}$ & & & & & $\mathrm{x}$ & $\mathrm{x}$ & & & & & \\
\hline Saberi, 2020 & & $\mathrm{x}$ & & & & & & & & & & & & & & & & & & & & \\
\hline Frecuencia & 1 & 9 & 4 & 1 & 3 & 3 & 2 & 11 & 2 & 3 & 8 & 9 & 5 & 7 & 5 & 7 & 8 & 2 & 3 & 1 & 1 & 2 \\
\hline
\end{tabular}

Continuación Tabla 2

Facilitadores. A nivel administrativo, los facilitadores que fueron destacados por la literatura fueron la flexibilidad en el proceso de consentimiento informado y la estandarización de los protocolos para los comités de ética en catorce $(11,13,15-17,19,20,23,25,28,30-33)$ y trece estudios $(12,13,17,20,21,24-26,28-30,32,34)$, respectiva- mente. En cuanto a los aspectos organizacionales y de logística, los documentos reportaron un alto número de facilitadores, sobresaliendo la adopción de soluciones virtuales al proceso de reclutamiento, recolección de datos, conducción y seguimiento en dieciséis estudios $(11,13-17,19,20,22,27-32,34)$ y la cooperación entre investigadores, patrocinadores 
y entidades de regulación en quince documentos $(6,11-14,16,18,20-22,24,29,32,33,35)$. A nivel metodológico, el facilitador que más se reportó fue la adopción de diseños flexibles que se puedan adaptar a las necesidades que impone la pandemia $(6,16,20,32)$. Se encontró que la categoría en que menos se reportaron datos fue en la asociada a la estructura sociocultural, donde se mencionan facilitadores como el diálogo con potenciales participantes $(33,34)$ y las publicaciones orientadas a la apropiación social del conocimiento $(13,35)$-ver Tabla 3-.

\begin{tabular}{|c|c|c|c|c|c|c|c|c|c|c|c|c|c|c|c|c|c|}
\hline \multirow[t]{2}{*}{$\begin{array}{c}\text { Referencia/ } \\
\text { Barrera }\end{array}$} & \multicolumn{5}{|c|}{$\begin{array}{l}\text { "Facilitadores } \\
\text { asociados a la } \\
\text { estructura social" }\end{array}$} & \multicolumn{2}{|c|}{$\begin{array}{l}\text { Facilitadores } \\
\text { asociadas } \\
\text { a trámites } \\
\text { administrativos }\end{array}$} & \multicolumn{7}{|c|}{$\begin{array}{c}\text { "Facilitadores organizacionales } \\
\text { asociados a la logística del } \\
\text { estudio" }\end{array}$} & \multicolumn{3}{|c|}{$\begin{array}{l}\text { Facilitadores } \\
\text { metodológicos }\end{array}$} \\
\hline & 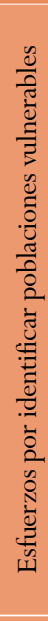 & 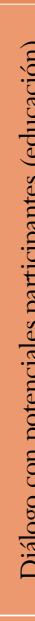 & & 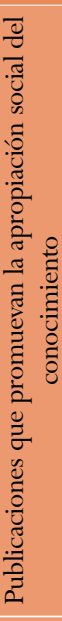 & 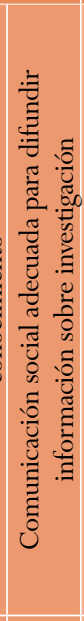 & 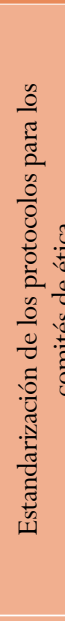 & - & 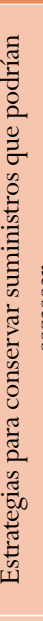 & 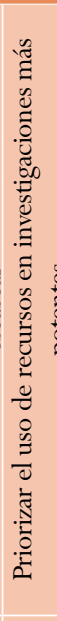 & 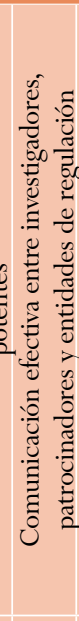 & 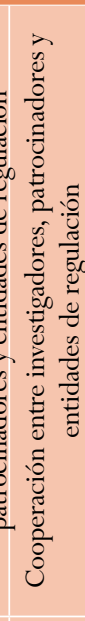 & 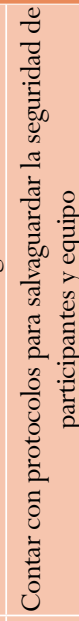 & 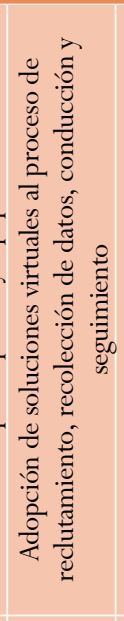 & 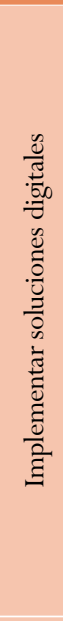 & 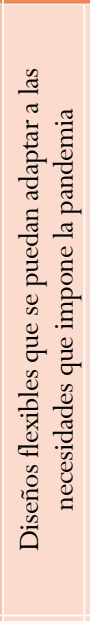 & 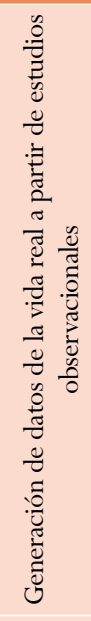 & 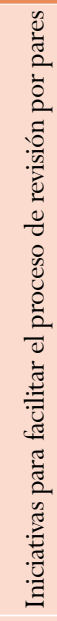 \\
\hline $\begin{array}{c}\text { Hashem et al, } \\
2020\end{array}$ & & & & & & $\mathrm{x}$ & $\mathrm{x}$ & & & $\mathrm{x}$ & $\mathrm{x}$ & & $\mathrm{x}$ & & $\mathrm{x}$ & & \\
\hline $\begin{array}{c}\text { Madariaga et } \\
\text { al, } 2021\end{array}$ & & & & & & $\mathrm{x}$ & $\mathrm{x}$ & & & & $\mathrm{x}$ & & $\mathrm{x}$ & $\mathrm{x}$ & $\mathrm{X}$ & & \\
\hline $\begin{array}{c}\text { Verna et al, } \\
2020\end{array}$ & & & & & & & $\mathrm{x}$ & & & & $\mathrm{x}$ & $x$ & $\mathrm{x}$ & & & & \\
\hline $\begin{array}{c}\text { AlNaamani et } \\
\text { al, } 2020\end{array}$ & & & & & & $\mathrm{x}$ & & & & & $\mathrm{x}$ & & & & & $\mathrm{x}$ & \\
\hline $\begin{array}{c}\text { Cagnazzo et } \\
\text { al, } 2021\end{array}$ & & & & & & $\mathrm{x}$ & $\mathrm{x}$ & & & & & & $\mathrm{x}$ & $\mathrm{x}$ & & & \\
\hline $\begin{array}{c}\text { Carracedo et } \\
\text { al, } 2021\end{array}$ & & & & & & & & & & & $\mathrm{x}$ & $\mathrm{x}$ & & & $\mathrm{x}$ & & \\
\hline $\begin{array}{l}\text { González- } \\
\text { Duarte et al, } \\
2020\end{array}$ & $\mathrm{x}$ & & & & & $\mathrm{x}$ & $\mathrm{x}$ & & & & & & & $\mathrm{x}$ & & & \\
\hline $\begin{array}{c}\text { Meyer et al, } \\
2021\end{array}$ & & & & & & $\mathrm{x}$ & & $\mathrm{x}$ & $\mathrm{x}$ & & $\mathrm{x}$ & & & & & & \\
\hline $\begin{array}{c}\text { Dron et al, } \\
2021\end{array}$ & & & & & & & & & & & $\mathrm{x}$ & & $\mathrm{x}$ & & & & \\
\hline $\begin{array}{c}\text { Bahans et al } \\
2021\end{array}$ & & & & & & $\mathrm{x}$ & & & & & & & & & & & \\
\hline $\begin{array}{c}\text { Yang et al, } \\
2020\end{array}$ & & & & & & & & & & & & & & & & & \\
\hline
\end{tabular}




\begin{tabular}{|c|c|c|c|c|c|c|c|c|c|c|c|c|c|c|c|c|c|c|}
\hline \multirow[t]{2}{*}{$\begin{array}{c}\text { Referencia/ } \\
\text { Barrera }\end{array}$} & \multicolumn{5}{|c|}{$\begin{array}{l}\text { "Facilitadores } \\
\text { asociados a la } \\
\text { estructura social" }\end{array}$} & \multicolumn{3}{|c|}{$\begin{array}{l}\text { Facilitadores } \\
\text { asociadas } \\
\text { a trámites } \\
\text { administrativos }\end{array}$} & \multicolumn{7}{|c|}{$\begin{array}{c}\text { "Facilitadores organizacionales } \\
\text { asociados a la logística del } \\
\text { estudio" }\end{array}$} & \multicolumn{3}{|c|}{$\begin{array}{l}\text { Facilitadores } \\
\text { metodológicos }\end{array}$} \\
\hline & 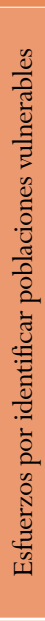 & 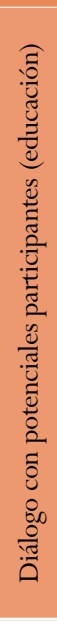 & 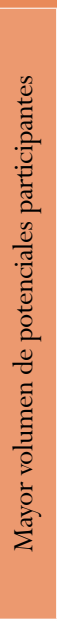 & 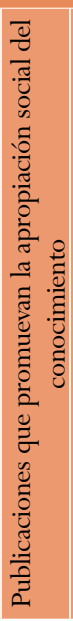 & 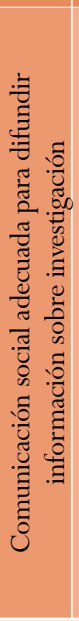 & 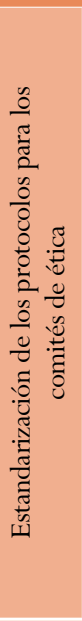 & 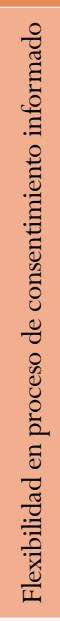 & 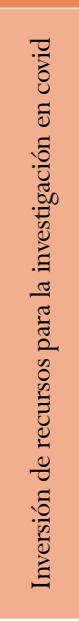 & 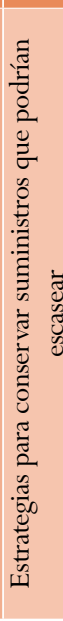 & 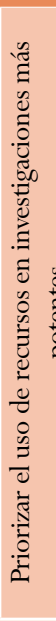 & 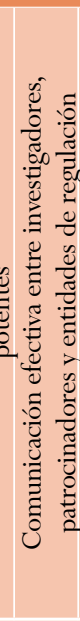 & 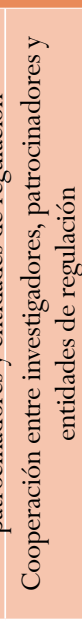 & 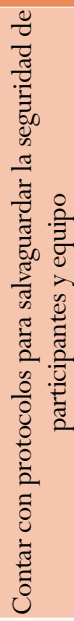 & 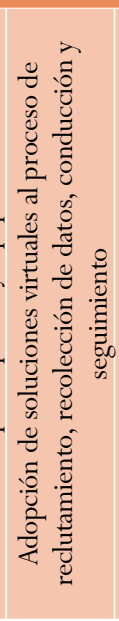 & 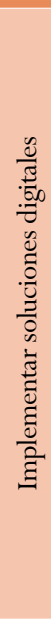 & 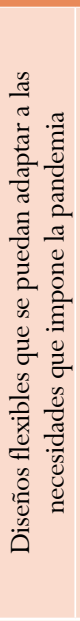 & 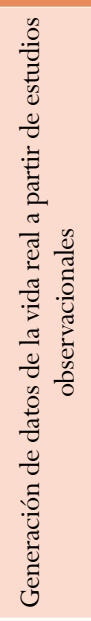 & 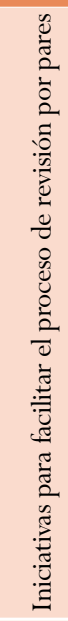 \\
\hline Bhatt 2020 & & & & & & & $\mathrm{x}$ & & & & $\mathrm{x}$ & & & $\mathrm{x}$ & & & & \\
\hline $\begin{array}{c}\text { Becker et al, } \\
2021\end{array}$ & & & & & & & & & & & & & & $\mathrm{x}$ & & & & \\
\hline $\begin{array}{c}\text { Ibrahim et al, } \\
2020\end{array}$ & & $\mathrm{x}$ & $\mathrm{x}$ & & $\mathrm{x}$ & & $\mathrm{x}$ & & & $\mathrm{x}$ & $\mathrm{x}$ & $\mathrm{x}$ & & & $\mathrm{x}$ & & & \\
\hline $\begin{array}{c}\text { Mourad et al, } \\
2020\end{array}$ & & & & $\mathrm{x}$ & & $\mathrm{x}$ & $\mathrm{x}$ & & $\mathrm{x}$ & $\mathrm{x}$ & $\mathrm{x}$ & $\mathrm{x}$ & & $\mathrm{x}$ & $\mathrm{x}$ & & & \\
\hline $\begin{array}{c}\text { Bierer et al, } \\
2020\end{array}$ & & & & & & & & & $\mathrm{x}$ & & & $\mathrm{x}$ & & $\mathrm{x}$ & & & & \\
\hline $\begin{array}{c}\text { Marcum et } \\
\text { al, } 2020\end{array}$ & & & & & & & $\mathrm{x}$ & & & & & & & $\mathrm{x}$ & $\mathrm{x}$ & & & \\
\hline $\begin{array}{c}\text { Almufleh et } \\
\text { al, } 2021\end{array}$ & & & & & & & $\mathrm{x}$ & & & & $\mathrm{x}$ & $\mathrm{x}$ & & $\mathrm{x}$ & & $\mathrm{x}$ & & \\
\hline $\begin{array}{c}\text { Sohrabi et al, } \\
2021\end{array}$ & & & & & & $\mathrm{x}$ & & $\mathrm{x}$ & & & $\mathrm{x}$ & $\mathrm{x}$ & & $\mathrm{x}$ & $\mathrm{x}$ & & & $\mathrm{x}$ \\
\hline $\begin{array}{l}\text { Singh et al, } \\
2020\end{array}$ & & & & & & $\mathrm{x}$ & $\mathrm{x}$ & & & $\mathrm{x}$ & $\mathrm{x}$ & & & $\mathrm{x}$ & & & & \\
\hline $\begin{array}{l}\text { Ma et al, } \\
2020\end{array}$ & & & & & & $\mathrm{x}$ & $\mathrm{x}$ & & & & $\mathrm{x}$ & $\mathrm{x}$ & & & & & & \\
\hline $\begin{array}{c}\text { Perez et al, } \\
2020\end{array}$ & & & & & & $\mathrm{x}$ & $\mathrm{x}$ & & & & & & & $\mathrm{x}$ & $\mathrm{x}$ & & & \\
\hline $\begin{array}{c}\text { Weiner et al, } \\
2020\end{array}$ & & & & & & & & $\mathrm{x}$ & $\mathrm{x}$ & & & $\mathrm{x}$ & & & & & & \\
\hline $\begin{array}{c}\text { Pepper et al, } \\
2020\end{array}$ & & & & $\mathrm{x}$ & & & & & & & & $\mathrm{x}$ & & & & & & \\
\hline Reyes, 2020 & & $\mathrm{x}$ & & & & $\mathrm{x}$ & & & & & $\mathrm{x}$ & & & $\mathrm{x}$ & & & & \\
\hline Saberi, 2020 & & & & & $x$ & & $\mathrm{x}$ & & $x$ & & & & & $x$ & & & & \\
\hline Frecuencia & 1 & 2 & 1 & 2 & 2 & 13 & 14 & 2 & 5 & 4 & 8 & 15 & 2 & 16 & 8 & 4 & 1 & 1 \\
\hline
\end{tabular}

Continuación Tabla 3 
Iniciativas y directrices difundidas por algunas autoridades en salud e investigación. Se identificaron documentos de las siguientes organizaciones: Cochrane (37), los Centros para el Control y la Prevención de Enfermedades (CDC) (38), la Organización para la Cooperación y el Desarrollo Económicos (OCDE) (39) y la OMS (40).

Se identificaron 3 temas principales: estrategias de priorización $(37,38,40)$ y enfoques inclusivos en la investigación (39), estrategias para facilitar la cooperación entre instituciones $(38,39)$, y estrategias para fortalecer la calidad metodológica de los estudios (37-40) y combatir la infodemia $(37,39,40)$. Estrategias de priorización y enfoques inclusivos en la investigación. El CDC, Cochrane y la OMS publicaron una serie de temáticas para orientar a los investigadores sobre los vacíos del conocimiento en diferentes aspectos alrededor de la pandemia. Se busca fomentar los estudios con resultados socialmente relevantes y que informen a los tomadores de decisiones de diversa naturaleza. Un ejemplo de esto es el "Cochrane COVID Review response", que detalla cómo se ha trabajado en la priorización de preguntas de investigación útiles y pertinentes, creando un banco de preguntas que lleva registro del trabajo investigativo desde que inició la pandemia (37). Por su parte, la CDC publicó la "CDC Science Agenda for COVID-19, 2020-2023”, en donde señala seis áreas prioritarias para la investigación sobre Covid (38), la OMS ha establecido una plataforma virtual en su página web dedicada a la comunicación oficial de respuestas a preguntas frecuentes (40).

Por otra parte, el CDC, en su agenda para la ciencia, hace énfasis en la importancia de tener un enfoque inclusivo en la investigación que tenga en cuenta la relación entre la inequidad social y el impacto de la pandemia (38). Para esto, buscan fomentar el uso de abordajes y metodologías cultural y lingüísticamente sensibles, así como la inclusión activa de poblaciones en situación de vulnerabilidad que se encuentran en riesgo de mayores dificultades a raíz del Covid-19. En cuanto a la relevancia social del conocimiento, la OCDE ha alentado la prepublicación de estudios importantes en diversos servidores diseñados para esto, como un medio para lograr la difusión rápida y oportuna del conocimiento (39).

Estrategias para facilitar la cooperación entre instituciones. El CDC propone la colaboración con países y organizaciones sociales para desarrollar la capacidad de investigación (37). La OCDE, por su parte, establece desafíos como la coordinación e interoperabilidad para acelerar el ritmo de la investigación y mejorar su calidad. Propone como mecanismo clave las políticas de ciencia abierta para la libre circulación de datos e ideas de investigación (38). Además, la OCDE comparte el compromiso de 117 organizaciones de proporcionar acceso abierto inmediato a publicaciones revisadas por homólogos, comunicar los resultados mediante servidores de prepublicación y compartirlos con la OMS (39). La OCDE estableció dentro de las acciones puntuales para fortalecer la respuesta a la crisis el asegurarse de contar con capital humano y recursos materiales adecuados para gestionar estudios de calidad (39).

Estrategias para fortalecer la calidad metodológica de los estudios y combatir la infodemia. Cochrane y la OMS (41) han buscado dar un manejo adecuado de la información, con el objetivo de que la sociedad consuma el conocimiento de manera responsable por medio de canales oficiales de comunicación accesibles al público teniendo un impacto positivo en estas comunidades y, así, disminuya la desinformación y las noticias falsas. Para ello estableció el "COVID-19 living evidence project” (42), un espacio en su plataforma electrónica en el cual los tomadores de decisiones pueden acceder a información de ensayos clínicos sobre Covid en el cual se evalúa la validez de los resultados en un formato sencillo y que son frecuentemente actualizados por investigadores independientes que tienen experiencia en síntesis de evidencia.

\section{DISCUSIÓN}

Resumen de resultados más importantes. En esta revisión se logró determinar las barreras y los facilitadores más comúnmente mencionados en la literatura 
dentro de cuatro grandes categorías: aspectos socioculturales, aspectos administrativos, aspectos organizacionales y de logística, y aspectos metodológicos. Además, es importante mencionar que estas categorías interactúan de manera compleja y que su relación no es unidireccional, puesto que se afectan entre ellas, como por ejemplo, los trámites administrativos dificultan la cooperación inter e intrainstitucional (16) o el aspecto del riesgo biológico de los investigadores de campo en cuanto a las implicaciones logísticas u organizacionales antes descritas. Las barreras mencionadas más frecuentemente en la literatura fueron las dificultades en cuanto al acceso a los participantes, que afectan los tamaños de muestra necesarios; las limitaciones de acceso a medios electrónicos para acceder desde diferentes contextos a los estudios; los trámites y las limitaciones de recursos de los comités de ética; el riesgo biológico y las limitaciones contractuales para los investigadores y la falta de coordinación inter e intrainstitucional. Los facilitadores identificados fueron la adopción de soluciones virtuales al proceso de reclutamiento, recolección de datos, conducción y seguimiento; la cooperación entre investigadores, patrocinadores y entidades de regulación; la flexibilidad en el proceso de obtención del consentimiento informado; y la utilización de metodologías adaptativas y publicaciones que facilitan la transferencia del conocimiento. Frente a las iniciativas difundidas por las autoridades en salud e investigación se identificaron cuatro estrategias de autoridades en salud e investigación para: a) priorización de preguntas de investigación, b) combatir la infodemia, c) fomentar la cooperación y la inclusión en la investigación, y d) para fortalecer la calidad metodológica de los estudios.

Amplitud de la búsqueda. Esta búsqueda hizo uso de las bases de datos Medline, Cochrane, Lilacs y Google Scholar de textos en inglés, español y portugués, factor que le dio amplitud a la búsqueda, ya que la mayoría de información sobre investigación en el contexto de pandemia estaba publicada en inglés. Por otra parte, la no inclusión de bases de datos como Embase, la no disponibilidad de térmi- nos MESH y DECS precisos para definir las barreras y los facilitadores en el contexto de la investigación en ciencias de la salud, así como la especificidad y novedad del tema de la revisión pudo haber afectado la identificación de algunos documentos y la diversidad de la búsqueda. No obstante, hemos incluido parte importante de información disponible hasta mayo de 2021.

Aplicabilidad de los resultados. El proceso de análisis de la literatura incluida en esta revisión de alcance permite poner en diálogo las barreras/facilitadores identificados por los investigadores durante la crisis desencadenada por la pandemia de Covid-19 y las iniciativas y directrices difundidas por algunas de las autoridades en salud e investigación. Este diálogo permite hacer una aproximación a ver cómo las inactivas desarrolladas llevan a superar las barreras y potenciar los facilitadores de la investigación en ciencias de la salud en el contexto académico, social y político actual.

Prioridades en la concepción, diseño, conducción y diseminación de la investigación: no solo se deben priorizar las preguntas a investigar, también aspectos relacionados con la conducción y la diseminación de la investigación. Respecto a este último aspecto, las iniciativas mencionadas por el CDC, Cochrane y el OCDE, que buscan que los resultados de los estudios tengan libre circulación y rápida difusión entre los tomadores de decisiones, concuerdan con algunas de las barreras identificadas en relación con aspectos socioculturales asociados a la investigación (ver Tabla 1), en donde se ha evidenciado el exceso de estudios repetidos, y la rápida perdida de vigencia de las investigaciones por los cambios en el comportamiento de la pandemia, tales como las nuevas variantes, los picos de contagios o periodos valle entre picos, y el acelerado ritmo de contagio de la infección $(21,25)$.

En cuanto a la conducción de los estudios, son importantes los aspectos organizacionales para protección de los derechos laborales y protección del riesgo biológico que se relacionan estrechamente con las recomendaciones que ofrece la OCDE sobre 
la disponibilidad y preservación del capital humano en los estudios. Otro aspecto en la conducción de los estudios son procedimientos de consentimiento informado, el seguimiento y el registro de la información $(13,20,29)$. La FDA (43) publicó como recurso distintas guías y pautas para la transición a soluciones digitales, con énfasis en procesos como el consentimiento informado, la conducción, el seguimiento del estudio y el registro de la información. Estrategias para combatir la infodemia: por otra parte, las iniciativas también buscan garantizar que se pueda tener certeza de que la evidencia resultado de la investigación sea válida y de buena calidad, como medida de protección contra las noticias falsas que afectan la toma adecuada de decisiones. El problema de la infodemia es identificado como barrera por varios autores (ver Tabla 2) y es entendida como "una cantidad excesiva de información -en algunos casos correcta, en otros no- que dificulta que las personas encuentren fuentes confiables y orientación fidedigna cuando las necesitan" (44). Estos autores además señalan que cuando el público consume noticias y conocimiento de fuentes no oficiales, se generan procesos sociales que impactan la opinión pública frente a la respuesta al Covid-19 y las prácticas sociales alrededor de la prevención de contagio y el tratamiento de la infección. A su vez, esto facilita que se genere una desconfianza social en los expertos y en las autoridades competentes $(26,29,32)$. Por todo esto, Cochrane y la OMS llevan a cabo iniciativas con el fin de contribuir a la lucha contra la infodemia y los efectos sociales que esta trae consigo.

Los facilitadores que fueron identificados en los estudios revisados, como la producción de publicaciones que faciliten la apropiación social del conocimiento y nuevas estrategias para difundirlas $(13,19,33,35)$, se alinean con las iniciativas planteadas por estas entidades. Ibrahim et al. (33), así como Saberi (19), sugieren que una buena comunicación con los medios y estrategias de educación para la población facilita el proceso investigativo en todas sus etapas.
Enfoque inclusivo de investigación: otro punto de encuentro es la necesidad de producir investigación sensible a los contextos y las diferentes poblaciones. Muchas de las barreras de acceso identificadas por Verna et al. están directamente relacionadas con los índices de pobreza y la inequidad de los países y, por esta razón, es común que las poblaciones más vulnerables, que con frecuencia son impactadas de manera diferencial tanto por la infección como por la crisis socioeconómica que desencadena, son excluidas también de los potenciales beneficios que trae participar en una investigación en ciencias de la salud. Como se mencionó anteriormente, el CDC postula como un asunto central la adopción de un enfoque inclusivo para combatir esta desigualdad a nivel local (participantes de diversos contextos) y a nivel internacional (colaboración con países en desarrollo) (38).

Estrategias para facilitar la cooperación entre instituciones: otro punto de gran importancia que resalta en las agendas de investigación de la OCDE y la OMS a nivel global es la comunicación efectiva y la colaboración internacional entre investigadores, patrocinadores y entidades de regulación. Al realizar esta revisión de alcance, llama especialmente la atención que los documentos coinciden con las autoridades en salud e investigación en la relevancia de la cooperación global para la investigación en ciencias de la salud en medio de la pandemia, así como el establecimiento de un clima académico de colaboración en vez de competencia $(14,18,29)$, y proponen la publicación de acceso abierto como mecanismo para conducir a un desarrollo más rápido de soluciones para problemas urgentes (35).

Nuestra revisión tiene algunas limitaciones: teniendo en cuenta que el problema de investigación continúa siendo un tema foco de la actualidad, es posible que exista nueva información publicada después de la fecha de búsqueda que no se haya detectado para esta revisión de alcance. Finalmente, los documentos encontrados presentan una alta variabilidad, por lo cual no se evaluó la calidad de estos. 


\section{CONCLUSIONES}

Para la investigación en el contexto de la pandemia representa un desafío continuar con la cooperación e interoperabilidad entre las instituciones, los países y las disciplinas en el futuro para facilitar los procesos investigativos, y cobra importancia mantener la ciencia abierta y la financiación de estudios cooperativos cuando surjan otras prioridades. Asimismo, es evidente la necesidad de desarrollar y sostener mecanismos para gestionar la información de manera eficiente para la toma de decisiones. Se requiere evaluar de manera continua los efectos que ha dejado esta pandemia en la práctica de la investigación en ciencias de la salud para comprender de manera integral lo que debemos aprender como sociedad a partir de las crisis.

\section{REFERENCIAS}

1. Organización mundial de la Salud. Coronavirus disease 2019 (COVID-19) Situation Report - 51. OMS; 2020. Disponible en: https://apps.who.int/iris/bitstream/ handle/10665/331475/nCoVsitrep11Mar2020-eng. pdf? sequence $=1$ \&isAllowed $=y$

2. World Health Organization. WHO Director-General's remarks at the media briefing on 2019-nCoV on 11 February 2020. WHO; 2020. Disponible en: https:/ www.who.int/es/director-general/speeches/detail/ who-director-general-s-remarks-at-the-media-briefingon-2019-ncov-on-11-february-2020.

3. The Economist. Covid-19 data: The pandemic's true death toll. 2021. Disponible en: https:/www.economist. com/graphic-detail/coronavirus-excess-deaths-estimates.

4. Banco Mundial. La COVID-19 (coronavirus) hunde a la economía mundial en la peor recesión desde la Segunda Guerra Mundial. 2020. Disponible en: https://www. bancomundial.org/es/news/press-release/2020/06/08/ covid-19-to-plunge-global-economy-into-worst-recession-since-world-war-ii

5. Organización Mundial de la Salud. World experts and funders set priorities for COVID-19 research. OMS; 2020. Disponible en: https://www.who.int/news/item/1202-2020-world-experts-and-funders-set-prioritiesfor-covid-19-research
6. Carracedo S, Palmero A, Neil M, Hasan-Granier A, Saenz C, Reveiz L. The landscape of COVID-19 clinical trials in Latin America and the Caribbean: assessment and challenges. Rev Panam Salud Pública. 2020; 44:1 5. https://doi.org/10.26633/RPSP.2020.177

7. UNESCO. R\&D Spending by country. 2021. Disponible en: http://uis.unesco.org/apps/visualisations/ research-and-development-spending/

8. Peters MDJ, Godfrey C, McInerney P, Baldini Soares C, Khalil H, Parker D. Chapter 11: Scoping Reviews. In: Aromataris E, Munn Z, editors. Joanna Briggs Institute Reviewer's Manual. The Joanna Briggs Institute; 2017. Disponible en: https://reviewersmanual.joannabriggs. org/

9. Ouzzani M, Hammady H, Fedorowicz Z, Elmagarmid A. Rayyan — a web and mobile app for systematic reviews. Syst Rev. 2016; 5(1):210. https://doi. org/10.1186/s13643-016-0384-4

10. Tricco AC, Lillie E, Zarin W, O'Brien KK, Colquhoun H, Levac D, et al. PRISMA Extension for Scoping Reviews (PRISMA-ScR): Checklist and Explanation. Ann Intern Med. 2018;169(7):467-73. https://doi. org/10.7326/M18-0850

11. Verna EC, Serper M, Chu J, Corey K, Fix OK, Hoyt $\mathrm{K}$, et al. Clinical Research in Hepatology in the COVID-19 Pandemic and Post-Pandemic Era: Challenges and the Need for Innovation. Hepatology. 2020; 72(5):1819-37. https://doi.org/10.1002/hep.31491

12. Meyer MN, Gelinas L, Bierer BE, Hull SC, Joffe S, Magnus D, et al. An ethics framework for consolidating and prioritizing COVID-19 clinical trials. Clin Trials. 2021; 18(2):226-33. https://doi. org/10.1177/1740774520988669

13. Mourad M, Bousleiman S, Wapner R, Gyamfi-Bannerman C. Conducting research during the COVID-19 pandemic. Semin Perinatol. 2020; 44(7):151287. https://doi.org/10.1016/j.semperi.2020.151287

14. Bierer BE, White SA, Barnes JM, Gelinas L. Ethical Challenges in Clinical Research During the COVID-19 Pandemic. J Bioethical Inq. 2020; 17(4):717-22. https://doi.org/10.1007/s1 1673-020-10045-4

15. Marcum M, Kurtzweil N, Vollmer C, Schmid L, Vollmer A, Kastl A, et al. COVID-19 pandemic and impact 
on cancer clinical trials: An academic medical center perspective. Cancer Med. 2020; 9(17):6141-6. https:// doi.org/10.1002/cam4.3292

16. Almufleh A, Joseph J. The time is now: role of pragmatic clinical trials in guiding response to global pandemics. Trials. 2021; 22(1):229. https://doi.org/10.1186/ s13063-021-05165-0

17. Perez T, Perez RL, Roman J. Conducting Clinical Research in the Era of Covid-19. Am J Med Sci. 2020; 360(3):213-5. https://doi.org/10.1016/j.amjms.2020.06.011

18. Weiner DL, Balasubramaniam V, Shah SI, Javier JR, on behalf of the Pediatric Policy Council. COVID-19 impact on research, lessons learned from COVID-19 research, implications for pediatric research. Pediatr Res. 2020; 88(2):148-50. https://doi.org/10.1038/ s41390-020-1006-3

19. Saberi P. Research in the Time of Coronavirus: Continuing Ongoing Studies in the Midst of the COVID-19 Pandemic. AIDS Behav. 2020; 24(8):2232-5. https:// doi.org/10.1007/s10461-020-02868-4

20. Madariaga A, Kasherman L, Karakasis K, Degendorfer P, Heesters AM, Xu W, et al. Optimizing clinical research procedures in public health emergencies. Med Res Rev. 2021; 41(2):725-38. https://doi.org/10.1002/ med. 21749

21. AlNaamani K, AlSinani S, Barkun AN. Medical research during the COVID-19 pandemic. World J Clin Cases. 2020; 8(15):3156-63. https://doi.org/10.12998/wjcc. v8.i15.3156

22. Dron L, Dillman A, Zoratti MJ, Haggstrom J, Mills EJ, Park JJH. Clinical Trial Data Sharing for COVID-19Related Research. J Med Internet Res. 2021; 23(3): e26718. https://doi.org/10.2196/26718

23. Yang Y, Li W, Zhang L, Zhang Q, Cheung T, Hall BJ, et al. The Impact of COVID-19 Pandemic on Clinical Research in China: Challenges and Progress. Front Med. 2020; 7:523. https://doi.org/10.3389/ fmed.2020.00523

24. Ma X, Wang Y, Gao T, He Q, He Y, Yue R, et al. Challenges and strategies to research ethics in conducting COVID-19 research. J Evid-Based Med. 2020; 13(2):173-7. https://doi.org/10.1111/jebm.12388
25. González-Duarte A, Kaufer-Horwitz M, Aguilar-Salinas CA. Bioethics in the COVID-19 Pandemic Research: Challenges and Strategies. Rev Investig Clínica. 2020; 72(5):4664. https://doi.org/10.24875/RIC.20000258

26. Bahans C, Leymarie S, Malauzat D, Girard M, Demiot C. Ethical considerations of the dynamics of clinical trials in an epidemic context: Studies on COVID-19. Ethics Med Public Health. 2021; 16:100621. https:// doi.org/10.1016/j.jemep.2020.100621

27. Becker G, Martin T, Sabo AN, Bertrand F, Hutt A, Ayme-Dietrich E, et al. Impact of the COVID-19 pandemic on clinical research in hospitals: observational study in the first epicenter of the epidemic during the general lockdown in France. Eur Rev Med Pharmacol Sci. 2021; 25(2):1158-1162. https://doi. org/10.26355/eurrev_202101_24686

28. Cagnazzo C, Besse M-G, Manfellotto D, Minghetti P, Cazzaniga S, Cottini L, et al. Lessons learned from COVID-19 for clinical research operations in Italy: what have we learned and what can we apply in the future? Tumori J. 2021; 107(1):6-11. https://doi. org/10.1177/0300891620977916

29. Sohrabi C, Mathew G, Franchi T, Kerwan A, Griffin $\mathrm{M}, \mathrm{C}$ Del Mundo JS, et al. Impact of the coronavirus (COVID-19) pandemic on scientific research and implications for clinical academic training - A review. Int J Surg. 2021; 86:57-63. https://doi.org/10.1016/j. ijsu.2020.12.008

30. Singh JA, Bandewar SV, Bukusi EA. The impact of the COVID-19 pandemic response on other health research. Bull World Health Organ. 2020;98(9):62531. https://doi.org/10.2471/BLT.20.257485

31. Bhatt A. Clinical trials during the COVID-19 pandemic: Challenges of putting scientific and ethical principles into practice. Perspect Clin Res. 2020; 11(2):59. https://doi.org/10.4103/picr.PICR_77_20

32. Hashem H, Abufaraj M, Tbakhi A, Sultan I. Obstacles and Considerations Related to Clinical Trial Research During the COVID-19 Pandemic. Front Med. 2020; 7:598038. https://doi.org/10.3389/ fmed.2020.598038

33. Ibrahim H, Kamour AM, Harhara T, Gaba WH, Nair SC. Covid-19 pandemic research opportunity: Is the 
Middle East \& North Africa (MENA) missing out? Contemp Clin Trials. 2020; 96:106106. https://doi. org/10.1016/j.cct.2020.106106

34. Reyes M. Research in the Time of COVID-19: Challenges of Research Ethics Committees. J ASEAN Fed Endocr Soc. 2020;35(1):29-32. https://doi. org/10.15605/jafes.035.01.07

35. Pepper MS, ChB M, Burton SG. Research in COVID-19 times: The way forward. In Pract. 2020;110(8):3.

36. Caicedo-Rosero ÁV, Estrada-Montoya JH. Barreras geográficas de acceso a los servicios de salud oral en el departamento de Nariño, Colombia. Rev Gerenc Polit Salud. 2016;15(31): 146-174. https://doi. org/10.11144/Javeriana.rgyps15-31.bgas

37. Cochrane. Coronavirus (COVID-19) - Cochrane resources and news. 2021. Disponible en: https:// www.cochrane.org/our-evidence/coronavirus-covid19-resources

38. Centers for Disease Control and Prevention. CDC Science Agenda for COVID-19, 2020-2023. Building the Evidence Base for Ongoing COVID-19 Response. CDC; 2021. Disponible en: https://www.cdc.gov/ coronavirus/2019-ncov/science/science-agendacovid 19.html\# print

39. Organización para la Cooperación y el Desarrollo Económicos. Medidas políticas clave de la OCDE ante el coronavirus (COVID-19): Por qué la ciencia abierta es esencial para combatir el COVID-19. OECD; 2020. Disponible en: https://www.oecd.org/coronavirus/policy-responses/por-que-la-ciencia-abierta-esesencial-para-combatir-el-covid-19-f3b83813/

40. Organización Mundial de la Salud. Coronavirus disease (COVID-19) pandemic. 2021; Disponible en: https:/www.who.int/emergencies/diseases/novelcoronavirus-2019

41. World Health Organization, Cochrane. The COVIDNMA initiative: A living mapping and living systematic review of Covid-19 trials. 2021. Disponible en: https:// covid-nma.com/

42. Boutron I, Chaimani A, Meerpohl JJ, Hróbjartsson A, Devane D, Rada G, et al. The COVID-NMA Project: Building an Evidence Ecosystem for the COVID-19 Pandemic. Ann Intern Med. 2020;173(12):1015-7. https://doi.org/10.7326/M20-5261

43. Food and Drug Adminstration. FDA Guidance on Conduct of Clinical Trials of Medical Products during COVID-19 Pandemic. Guidance for Industry, Investigators, and Institutional Review Boards [Updated on March 21]. FDA; 2020. Disponible en: https:// www.fda.gov/regulatory-information/search-fdaguidance-documents/fda-guidance-conduct-clinicaltrials-medical-products-during-covid-19-pandemic

44. Pan American Health Organization. Entender la infodemia y la desinformación en la lucha contra la COVID-19. PAHO; 2020. Disponible en: https:// iris.paho.org/bitstream/handle/10665.2/52053/ Factsheet-Infodemic_spa.pdf?sequence $=16$

\section{FINANCIACIÓN}

Vicerrectoría de investigación, Universidad Nacional de Colombia.

\section{CONTRIBUCIÓN DE LAS AUTORAS}

Karen Daniela Neira-Fernández: concepción del artículo, recolección de datos, redacción y aprobación de la versión final.

Laura Gaitán-Lee: concepción del artículo, recolección de datos, redacción y aprobación de la versión final.

Olga Janneth Gómez-Ramírez: concepción del artículo, recolección de datos, redacción, revisión crítica del artículo, y aprobación de la versión final. 